\title{
Indicadores de Inovação no Plano de Desenvolvimento Institucional do IF Goiano
}

\author{
Innovation Indicators in the Institutional Development Plan of IF Goiano
}

\author{
Bruna de Oliveira Santos ${ }^{1}$ \\ Paulo Gustavo Barboni Dantas Nascimento ${ }^{2}$ \\ ${ }^{1}$ Instituto Federal Goiano, Goiânia, GO, Brasil \\ ${ }^{2}$ Universidade de Brasília, Brasília, DF, Brasil
}

\begin{abstract}
Resumo
Este artigo tem como objetivo criar indicadores de inovação no Plano de Desenvolvimento Institucional (PDI) que atendam ao propósito e de estruturar e dar competências suficientes para sensibilizar, disseminar e implementar uma cultura inovadora no IF Goiano, aprimorando, assim proteção do capital intangível e a transferência de tecnologia. A pesquisa foi norteada pelos fundamentos do método da pesquisa-ação, pesquisa documental $e$ uso da ferramenta de benchmarking para identificar estratégias utilizadas por outras instituições e técnicas de bibliometria com o propósito de elaborar indicadores personalizados. O IF Goiano tem potencial inovador e o aproveitamento desse potencial, associado a ações de gestão da inovação, como inserção de indicadores de inovação no PDI, ao uso de roadmap para definir linhas de pesquisa, à formulação de editais com critérios de produtividade tecnológica $e$ inovadora $e$ a atividades similares, tende a fortalecer a cultura de inovação para que a instituição atinja maior maturidade de inovação.
\end{abstract}

Palavras-chave: Indicadores de Inovação. Planejamento Estratégico. Propriedade Intelectual.

\begin{abstract}
The objective was to create innovation indicators in the Institutional Development Plan (IDP), which aims to structure and give sufficient skills to sensitize, disseminate and implement an innovative culture in IF Goiano, improving the protection of intangible capital and the transfer of technology. The research was guided by the fundamentals of the action-research method, documentary research and the use of the benchmarking tool to identify strategies used by other institutions and bibliometrics techniques to develop customized indicators. IF Goiano has innovative potential, using this potential allied to actions of innovation management: insertion of innovation indicators in the PDI, use of roadmap to define lines of research, formulation of edicts with criteria of technological and innovative productivity, and similar activities, tend to strengthen the culture of innovation so that the institution achieves greater maturity of innovation.
\end{abstract}

Keywords: Innovation Indicators. Strategic Planning. Intellectual Property.

Área Tecnológica: Administração Pública. Gestão da Inovação. 


\section{Introdução}

O Brasil passou na última década por mudanças no aspecto das políticas de suporte à inovação. Tais mudanças vieram por meio da Lei de Inovação, que regulamentou a atividade de pesquisadores de instituições públicas em projetos fomentados com empresas e autorizou a comercialização dos produtos tecnológicos, provenientes dessas parcerias.

A Lei do Bem também colaborou quando possibilitou maior abrangência e desburocratizou a aplicabilidade de incentivos fiscais para investimentos privados em Pesquisa e Desenvolvimento (P\&D).

Segundo estudo do Instituto de Pesquisa Econômica Aplicada (IPEA, 2017), ficou evidenciado que foram feitos investimentos significativos em Ciência e Tecnologia (C\&T), mas, de forma pulverizada, sem objetivos concretos de construir um sistema competitivo.

Parte dos esforços do governo refletiu na Rede Federal de Educação Profissional, Científica e Tecnológica (RFEPCT), criada pela Lei n. 11.892, de 29 de dezembro de 2008. No estado de Goiás, a RFEPCT é composta de IF Goiano e de IF Goiás. O IF Goiano, instituição jovem se comparada a grandes universidades, tem feito um trabalho significativo de oferta do ensino médio integrado profissionalizante e de cursos de graduação e pós-graduação. O estado de Goiás está inserido na Rede Centro Oeste de Inovação (Rede CO), que possui a missão de ampliar, promover e fortalecer o ambiente de inovação nos estados de Mato Grosso (MT), Mato Grosso do Sul (MS), Goiás (GO) e Distrito Federal (DF), interligando os principais agentes de inovação.

Todo esse ecossistema de inovação possui diversos atores, como as Instituições de Ciência e Tecnologia (ICTs), Incubadoras, Parques Tecnológicos, Startups, investidores, Fundações de Apoio, Empresa Brasileira de Pesquisa Agropecuária (Embrapa) e outros segmentos da área.

O ecossistema goiano de inovação possui agentes com potencial, como as instituições Universidade Federal de Goiás (UFG), Universidade Estadual de Goiás (UEG), Pontifícia Universidade Católica (PUC GO), Instituto Federal Goiano (IF Goiano), Instituto Federal de Goiás (IF Goiás), Gyntec, Rede Goiana de Inovação, Fundação de Amparo à Pesquisa do Estado de Goiás (FAPEG), Federação das Indústrias do Estado de Goiás (FIEG), Sistema S, Centro Universitário de Anápolis (UniEvangélica), entre outras faculdades particulares e empresas (principalmente do setor de agronegócios). Há registros de sete parques tecnológicos, um polo Embrapii e três clusters consolidados em Goiás que auxiliam no fortalecimento do sistema de inovação goiano. Esse ambiente de inovação oferece condições para que o IF Goiano se fortaleça e se torne mais produtivo.

Este estudo possui o objetivo de criar uma proposta de indicadores científicos, tecnológicos e de inovação que sejam capazes de avaliar e de fomentar a consolidação da política de inovação do IF Goiano.

A estratégia utilizada para desenvolver essa pesquisa foi constituída pelo mapeamento do contexto em que os constructos científicos e tecnológicos se desenvolvem no IF Goiano. Desse modo, foram utilizadas fundamentação teórica e técnicas de bibliometria e de benchmarking para sugerir indicadores de inovação no Plano de Desenvolvimento Institucional (PDI), além disso, também foram utilizados parâmetros de como avaliar o desempenho e promover ações que impulsionem a cultura da inovação e da pesquisa aplicada na instituição. 
Um estudo do Ipea (2017) menciona que são feitos muitos investimentos, principalmente em editais de fundos setoriais, com ausência de objetivos concretos de resolução de problemas, sendo que a grande maioria não tem o objetivo específico de fomentar a pesquisa aplicada. Tal situação faz com que os produtos desenvolvidos por tais editais não sejam aproveitados pelo setor público ou pela sociedade na forma de novas tecnologias ou produtos inovadores. Além disso, tais produtos não são acompanhados ou avaliados pelo agente financiador (do setor público) que sequer desenvolveu competências técnicas para avaliá-los (IPEA, 2017).

No relatório do Centro de Gestão e Estudos Estratégicos (CGEE, 2015), discute-se que não existe hegemonia dos indicadores de P\&D. A inexistência de indicadores de inovação consolidados e amplamente utilizados dificulta a implementação de políticas de inovação no Brasil. Por isso, é importante o esforço de desenvolvimento de indicadores de resultado do processo de inovação no país, como também é fundamental a avaliação dos resultados obtidos.

Dessa forma, a proposta de indicadores de inovação no PDI tem o intuito de mensurar as práticas de inovação desenvolvidas na instituição e incentivar sua maturidade. Esta pesquisa apresenta ainda a perspectiva de realizar, além de fazer a transferência de conhecimento, a utilização das tecnologias desenvolvidas nos projetos de PD\&I no mercado, contribuindo com o desenvolvimento regional e nacional.

\subsection{Inovação}

Segundo Schumpeter (1934), o processo dinâmico, no qual o desenvolvimento econômico é dirigido pelos impactos das inovações tecnológicas, denomina-se destruição criadora, que se caracteriza por novas tecnologias que substituem as antigas, contrariando a ideia de equilíbrio geral da economia, descrita na teoria neoclássica. Inovação não é apenas um termo técnico voltado para a tecnologia, pois se refere, também, às dimensões sociais e econômicas (SCHUMPETER, 1982). Para Drucker (1981), Van de Ven et al. (1999) e Toivonem e Tuominen (2009), a inovação é um sistema de desenvolvimento e de implantação de uma novidade, incluindo novos processos de desenvolvimento de ideias, novas tecnologias e novos produtos, arranjos ou processos.

O eixo chamado de inovação tem importância vital nesse cenário no qual o conhecimento possui valor intangível. As expansões dos mercados internos e externos, mundiais e locais, exigem precisão e definição da gestão inovadora da qual elas fazem parte.

Novos mercados são criados para produtos e serviços que impliquem uso intensivo de Tecnologia da Informação e Comunicação (TIC), e os bens e serviços de informação têm a característica de serem imateriais (TIGRE, 2006). O conhecimento ocupa um papel cada vez mais importante na economia, assumindo fatores de processos produtivos mais importantes do que terra, capital e trabalho.

Esse novo modo de gerir e de criar recursos tangíveis e intangíveis é um somatório de conhecimentos, recursos intelectuais, habilidades individuais ou de grupo, que, quando somados, criam recursos responsáveis pelo sucesso, crescimento e aumento do diferencial competitivo das organizações, garantindo, assim, sua sobrevida. 
Uma empresa possui vantagem competitiva, segundo Barney e Hesterly (2006), quando é capaz de gerar maior valor econômico do que suas concorrentes. Desse modo, as inovações são a principais iniciativas que uma organização pode realizar para garantir esse diferencial.

\subsection{Sistemas de Inovação (Tripla Hélice, Quádrupla Hélice e Quíntupla Hélice)}

As sociedades contemporâneas estão presenciando um processo de mudança na produção de conhecimento. $\mathrm{O}$ modelo tradicional de pesquisa tende a ser substituído por um novo, correspondente às operações processadas no interior de limites disciplinares, com barreiras nitidamente definidas que atuem dentro dos limites acadêmicos e descobertas científicas. O modelo da Tripla Hélice (TH), de Etzkowitz e Leydesdorff (1998), aponta continuidades no papel desempenhado pelas instituições que participam na produção desse sistema de conhecimento.

O foco principal não é o que elas desempenham, mas a relação basilar entre essas três instituições: a universidade, a indústria e o governo. No primeiro modelo, as três esferas (universidade, indústria e governo) são definidas institucionalmente. A interação entre elas ocorre por relações industriais, transferência de tecnologias e contratos oficiais, e são amplamente disseminadas em países desenvolvidos ou em fase de desenvolvimento. No segundo modelo, as hélices são definidas como diferentes sistemas de comunicação, que consistem em operações de mercado, inovação tecnológica e controle de interfaces. Tais interfaces geram novas formas de comunicação, ligadas à transferência de tecnologia, e constituem uma esfera intermediária. No terceiro modelo, as esferas institucionais da universidade, indústria e governo, com acréscimo às funções tradicionais, assumem papéis umas das outras. A universidade passa a ter uma função quase governamental, como disseminadora de conhecimento, inovação e tecnologia local e regional.

Com a evolução do mercado e rapidez das informações, o Sistema de Inovação passou por uma evolução conceitual, assim como o modelo de Tripla Hélice. O Estado atuava como ator principal, assim a academia e a indústria estavam submetidas à atuação deste. Porém, novas dinâmicas têm sido apresentadas, e a delimitação e influência de cada um desses atores sofreu alterações, com uma sobreposição e divisão equânime de papeis - em que todos partilham do mesmo tipo de influência, resultando assim em estruturas híbridas (CARAYANNIS; CAMPBELL, 2010). Essas estruturas híbridas englobam e derivam da Tripla Hélice, tornando-a mais abrangente com uma quarta e quinta hélices, com a formação de sistemas de inovação mais avançados.

No modelo da quádrupla hélice, a sociedade, como mostra a Figura 1, composta de pessoas e/ou usuários, incorpora-se como ator no ambiente de inovação. Já o modelo da quíntupla hélice caracteriza-se pelo conjunto das interações sociais e intercâmbios acadêmicos em um Estado (Estado-Nação). Nele, há o objetivo de promover e evidenciar um sistema cooperativo de conhecimentos, habilidades e inovações para um desenvolvimento mais sustentável (CARAYANNIS; CAMPBELL, 2010). 
Figura 1 - Modelos de quádrupla e quíntupla hélice
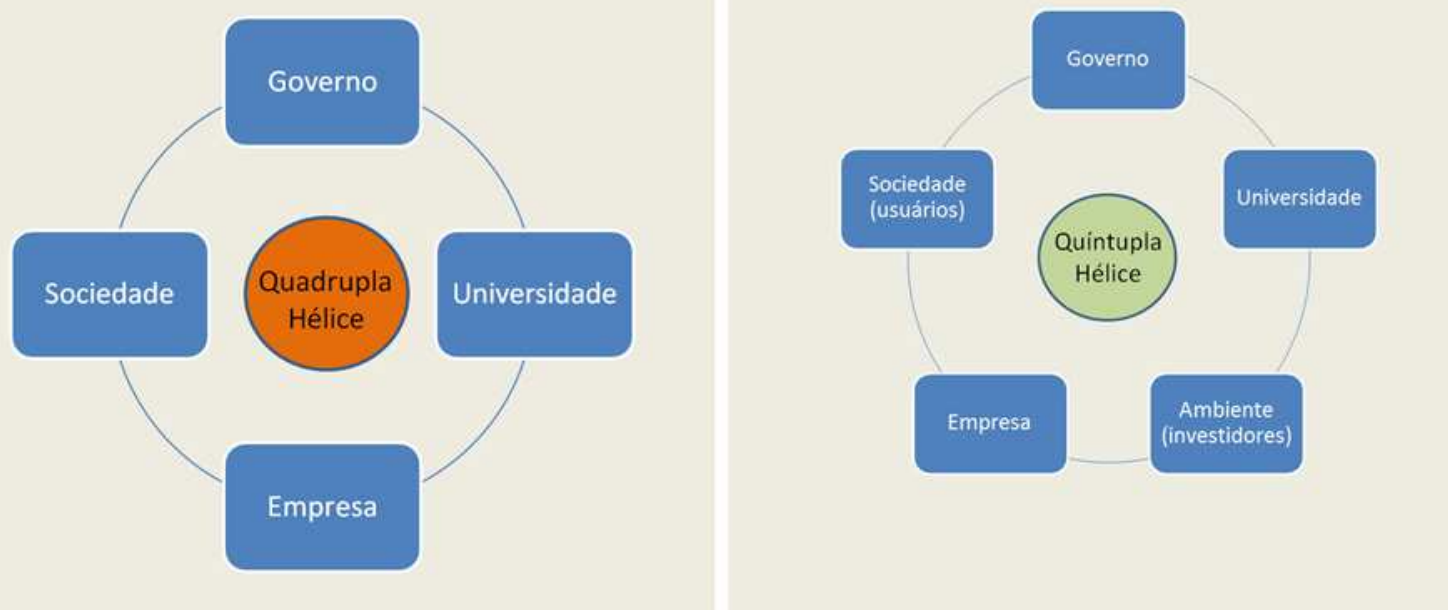

Fonte: Elaborada pelos autores deste artigo (2018)

\section{Indicadores de Ciência, Tecnologia e Inovação}

O uso de indicadores de Ciência, Tecnologia e Inovação (CT\&I) pode ser entendido como em constante e rápida evolução. De acordo com Freeman e Soete (2009), isso pode ser explicado por dois motivos: 1) pela facilidade de acesso ao ambiente informatizado: e 2) pelo interesse em um número crescente de políticas públicas e círculos de negócios privados envolvidos em tais indicadores.

No relatório da CGEE (2015) são colocadas três razões para a existência de indicadores de CT\&I:

Razão científica: analisa os fatores que influenciam a direção e a velocidade dos processos de expansão das fronteiras do conhecimento científico, verificando as relações existentes entre a pesquisa básica, pesquisa aplicada, desenvolvimento experimental $e$ inovação. Considera também os impactos de avanços tecnológicos na economia $e$ na sociedade, em geral, e em particular no emprego, na qualidade de vida e no meio ambiente;

Razão política: busca a formulação, avaliação e aperfeiçoamento de políticas, buscando o monitoramento da capacitação tecnológica de instituições, setores, regiões e/ou países. Procura fiscalizar a eficiência e eficácia de políticas, avaliando o desempenho, a qualidade e o potencial de instituições e grupos de pesquisa e de desenvolvimento envolvidos;

Razão pragmática: monitora tendências e perspectivas de evolução da CT\&I. A partir de indicadores, busca identificar as oportunidades tecnológicas, localizar as competências, monitorar o processo de mudança técnica, além de avaliar os impactos das mudanças tecnológicas.

Para Rosini e Palmisano (2003), todo sistema apresenta entradas de dados (input), processamento e saída das informações (output) e feedback. Os dados são os elementos que representam eventos ocorridos na empresa ou circunstâncias físicas, antes de serem organizados, de maneira que as pessoas possam entender e utilizar. 
O input é a coleta de dados internos ou externos na organização para que sejam processados; $e$, no processamento, ocorre a conversão ou tratamento da matéria-prima que assume outra forma para que o ser humano possa compreender; output é a saída e distribuição das informações processadas utilizadas para a tomada de decisão; e, finalmente, o feedback, no qual as informações fornecidas retornam para serem apropriadas e auxiliarem na avaliação ou correção do input.

A P\&D tem sido considerada como um esforço de inovação, sendo as patentes um dos principais mecanismos de apropriação de seus resultados. Apesar disso, é possível verificar problemas básicos com relação ao uso de indicadores que se referem ao assunto. Para Camillo et al. (2007), a variação no significado econômico das invenções, as diferenças setoriais e internacionais do processo de patenteamento e o peso idêntico atribuído a patentes de produtos que foram lançados no mercado e àquelas que são meramente invenções são alguns dos pontos mais discutidos na literatura.

É importante mencionar que o uso desses indicadores é considerado internacionalmente, destacando-se nesse âmbito três manuais utilizados para medição de índices de inovação, são eles: o Manual Frascati, o Manual de Oslo e o Manual de Bogotá.

Em relação ao Manual de Oslo, Stal e Campanário (2010) consideram que inovações resultam da área de produção, de controle de qualidade, ou de engenharia de produto e processo. A mensuração de CT\&I exige a avaliação de várias dimensões, o que gera a utilização de indicadores compostos.

Stal e Campanário (2010) alertam para as peculiaridades na mensuração em empresas, em que os dados podem ser questionados e não determinar automaticamente as decisões de investimentos e sua utilização em políticas públicas pela falta de modelos teóricos que norteiem a seleção e o peso dos diferentes indicadores em países distintos.

No Brasil, o uso de indicadores de CT\&I foi orientado pelo extinto Ministério do Planejamento, Orçamento e Gestão (MPOG), que, em 2010, elaborou um guia metodológico para construção de indicadores em programas do Governo Federal, sugerindo que os indicadores sejam construídos considerando as diferentes fases do ciclo de gestão: (a) insumo (input indicators); (b) processo (throughput indicators); (c) produto (output indicators), resultado (outcome indicators); e (d) impacto (impact indicators), os quais estão detalhados no Quadro 1.

Quadro 1 - Orientações do MPOG para construção de indicadores de CT\&I no Brasil

\begin{tabular}{|c|c|}
\hline FASE & DESCRIÇÃo \\
\hline $\begin{array}{c}\text { Insumo } \\
\text { (input indicators) }\end{array}$ & $\begin{array}{l}\text { São indicadores ex-ante facto que têm relação direta com os recursos a serem } \\
\text { alocados, ou seja, com a disponibilidade dos recursos humanos, materiais, } \\
\text { financeiros e outros a serem utilizados pelas ações de governo. Pode-se citar } \\
\text { como exemplos médicos/mil habitantes e gasto per capita com educação; }\end{array}$ \\
\hline $\begin{array}{c}\text { Processo } \\
\text { (throughput indicators) }\end{array}$ & $\begin{array}{l}\text { São medidas in curso ou intermediárias que traduzem o esforço empreendido } \\
\text { na obtenção dos resultados, ou seja, medem o nível de utilização dos } \\
\text { insumos alocados como, por exemplo, o percentual de atendimento de } \\
\text { um público-alvo e o percentual de liberação dos recursos financeiros; }\end{array}$ \\
\hline $\begin{array}{c}\text { Produto } \\
\text { (output indicators) }\end{array}$ & $\begin{array}{c}\text { Medem o alcance das metas físicas. São medidas ex-post facto que expressam as } \\
\text { entregas de produtos ou serviços ao público-alvo do Programa. São exemplos } \\
\text { o percentual de quilômetros de estrada entregues, de armazéns construídos } \\
\text { e de crianças vacinadas em relação às metas físicas estabelecidas; }\end{array}$ \\
\hline
\end{tabular}




\begin{tabular}{|c|c|c|}
\hline FASE & DESCRIção \\
\hline $\begin{array}{c}\text { Resultado } \\
\text { (outcome indicators) }\end{array}$ & $\begin{array}{c}\text { Essas medidas expressam, direta ou indiretamente, os benefícios no público-alvo } \\
\text { decorrentes das ações empreendidas no contexto do Programa e têm particular } \\
\text { importância no contexto de gestão pública orientada a resultados. São exemplos } \\
\text { as taxas de morbidade (doenças), taxa de reprovação escolar e de homicídios; }\end{array}$ \\
\hline $\begin{array}{c}\text { Impacto } \\
\text { (impact indicators) }\end{array}$ & $\begin{array}{c}\text { Possuem natureza abrangente e multidimensional, têm relação com a sociedade } \\
\text { como um todo e medem os efeitos das estratégias governamentais de médio e longo } \\
\text { prazos. Na maioria dos casos estão associados aos objetivos setoriais e de governo. }\end{array}$ \\
\hline
\end{tabular}

Fonte: Brasil (2010)

Ressalta-se, ainda, que os indicadores de CT\&I, em muitos casos, cumprem o propósito de aferir o desempenho das regiões ou dos países das instituições em ciência, tecnologia e inovação. Isso implica um benchmarking contínuo ou, pelo menos, uma comparação entre esses atores na dimensão de CT\&I.

Mesmo com os direcionamentos passados, verifica-se que os indicadores tradicionais $e$ os novos indicadores de CT\&I possuem suas limitações, diante das circunstâncias em que se encontra o processo de globalização. Diante disso, Velho (2001) destaca que apesar dos esforços direcionados pelo país [Brasil] para coletar informações, gerar bases de dados e produzir indicadores científicos e tecnológicos, ainda se está longe dos sistemas de informação em C\&T estabelecidos por países europeus.

Para Silva, Hartman e Reis (2008), é possível classificar as instituições de acordo com níveis de inovação, que são o Nível IN (inovadora), Nível MIN (mediana inovadora) e Nível PIN (pouco inovadora). O nível IN é quando a instituição apresenta um elevado nível de inovação tecnológica, não necessitando de muitos ajustes em seus processos de gestão para buscar um aumento de faturamento por meio de suas inovações. O nível MIN é quando a instituição tem nível médio de inovação tecnológica, necessitando alguns ou vários ajustes em seus processos de gestão para obter um maior faturamento pelas inovações tecnológicas. O nível PIN caracteriza-se pelo nível baixo de inovação tecnológica, necessitando muitos ajustes em seus processos de gestão para a obtenção de um faturamento oriundo de produtos, processos e/ou serviços inovadores.

Além de buscar parâmetros no atual estado da técnica sobre o tema, no processo de construção dos indicadores de inovação, teve-se a preocupação de eles estarem alinhados à missão do PDI do IF Goiano (2019), que consiste em "[...] promover educação profissional e tecnológica de excelência, visando à formação integral e emancipatória do cidadão para o desenvolvimento da sociedade". Nesse sentido, o estudo foi desenvolvido para compor o conjunto de ações para a realização da missão da ICT, provendo referências e boas práticas para o processo de elaboração da política de inovação institucional.

\section{Metodologia}

Para a Organization for Economic Co-operation and Development (OCDE, 2006), o entendimento dos aspectos referentes ao processo de inovação, como a interação entre os atores e os componentes envolvidos na pesquisa, é essencial para que sejam propostas políticas adequadas ao desenvolvimento da inovação. 
A pesquisa, descrita por este artigo, foi norteada pelos fundamentos do método da pesquisa-ação, na qual foi necessária uma investigação diagnóstica que compreendeu a análise de informações da pesquisa e permitiu a sugestão de ações que possam contribuir com melhores práticas de inovação no IF Goiano.

Para construir o referencial teórico foi utilizada uma pesquisa bibliográfica. Já a pesquisa documental permitiu conhecer e validar as informações dos indicadores. Os indicadores propostos foram estabelecidos após análise da estrutura organizacional do IF Goiano, definição de pesquisas prioritárias, política institucional e área de conhecimento predominante.

A obtenção de dados da pesquisa foi realizada por meio de pesquisa documental, impressa e divulgada eletronicamente, para tanto foi utilizado o recorte temporal de 2008 a 2018, com consulta a Relatórios de Gestão, base de dados Sistema Unificado de Administração Pública (SUAP), base de dados do Conselho Nacional de Desenvolvimento Científico e Tecnológico (CNPq), consulta pelo Sistema Eletrônico do Serviço de Informações ao Cidadão (e-SIC), relatórios do Instituto Nacional da Propriedade Industrial (INPI), entre outros documentos. A obtenção dos registros de artigos com Qualis foi realizada a partir de relatórios extraídos da Plataforma Carlos Chagas.

Utilizou-se, ainda, técnicas de bibliometria e a ferramenta de benchmarking para conhecer boas práticas de indicadores de inovação customizados e mencionados em outros planejamentos estratégicos de Institutos Federais (IF), como o do IFSC, IFES, IFPB, IFCE, para, a partir disso, elaborar o modelo aqui proposto, com indicadores que auxiliem e/ou sirvam para embasar discussões acerca da política de inovação do IF Goiano.

\section{Resultados e Discussão}

Este tópico privilegia a apresentação da instituição estudada, a análise dos resultados obtidos por meio do histórico organizacional e a correlação com o conhecimento extraído da literatura, ressaltando o nível de importância de cada indicador para o planejamento estratégico e as possíveis tendências, que podem potencializar o desenvolvimento da inovação no IF Goiano.

\subsection{IF Goiano}

O Instituto Federal de Educação, Ciência e Tecnologia Goiano (IF Goiano) foi criado por meio da Lei n. 11.892, de 29 de dezembro de 2008, que determinou que o Instituto Federal Goiano fosse vocacionado às ciências agrárias.

É uma instituição que oferece educação superior, básica e profissional, pluricurricular e multicampi, especializada em educação profissional e tecnológica. Em seus 12 campi, são ofertados diversos cursos que vão do ensino médio a cursos superiores de graduação, especialização, programas de mestrados e doutorados, além de cursos na modalidade Educação a Distância (EaD) e o Programa Nacional de Integração da Educação Profissional com a Educação Básica na Modalidade de Educação Jovens e Adultos (Proeja).

Em meados de 2014, o IF Goiano inaugurou o Polo de Inovação em Rio Verde. E, em 2017, o IF Goiano conquistou o credenciamento da Empresa Brasileira de Pesquisa e Inovação Industrial (Embrapii). O polo Embrapii de Tecnologias Agroindustriais (PETAgro) tem como 
missão gerar soluções inovadoras que melhorem a competitividade de parceiros agroindustriais (EMBRAPII, 2017), possui foco em processos tecnológicos para manejo agrícola e tecnologias para processamento e armazenamento de grãos. Em março de 2019, o IF Goiano conseguiu aprovar o projeto do Parque Científico Tecnológico, também instalado em Rio Verde e credenciado no Programa Goiano de Parques Tecnológicos (PGTec).

O NIT teve suas competências e obrigatoriedade instituídas na Lei de Inovação Tecnológica n. 10.973, de 2 de dezembro de 2004, alterada pela Lei n. 13.243, de 11 de janeiro de 2016, que estabeleceu a sua criação: estrutura instituída por uma ou mais Instituições Científicas, Tecnológicas e de Inovação (ICTs), com ou sem personalidade jurídica própria, que tenha por finalidade a gestão de política institucional de inovação. Devido a essa regulamentação ser tão recente, a maioria das instituições de ensino tem políticas de inovação em fase embrionária.

O modelo de planejamento estratégico do IF Goiano é participativo e tratado como ferramenta de gestão, denominado de Plano de Desenvolvimento Institucional (PDI).

O PDI, que é o "[...] documento em que se definem a missão, visão e valores da instituição e as estratégias para atingir metas e objetivos." (IF GOIANO, 2019). Abrange um período de cinco anos, o PDI 2019-2023 contemplará o cronograma e a metodologia de implementação dos objetivos, metas e ações do IF Goiano, observando a coerência e a articulação entre as diversas ações, a manutenção de padrões de qualidade.

A partir desse levantamento e do estabelecimento da missão, dos objetivos estratégicos e das metas do PDI do IF Goiano no período de 2019-2023, buscou-se criar indicadores de desempenho quantitativos alinhados a estratégia da instituição e que fossem objetivos. Foi utilizada a metodologia SMART - indicadores específicos (specific), mensuráveis (measurable), viáveis (attainable), relevantes (relevante) e temporizáveis (time-bound).

\subsection{Proposta de Indicadores de Inovação para o PDI do IF Goiano}

O IF Goiano apresenta em seu PDI 2014-2018 os seguintes indicadores de pesquisa: Taxa de Participação em Editais de Pesquisa (quantidade de participação em editais de pesquisa dividido pela quantidade de editais disponíveis nacionais e estaduais); Publicação de artigos científicos no exterior (total de artigos científicos publicados no exterior dividido pelo total de artigos científicos publicados); Taxa de alunos bolsistas (quantidade de bolsistas em pesquisa dividida pela quantidade de alunos matriculados). Desse modo, verifica-se no PDI 2014-2018 a ausência de indicadores de inovação, o que de certa forma até subestima a latente capacidade que o IF Goiano possui na área de pesquisa.

No novo mapa estratégico do PDI do IF Goiano é possível verificar que foi incluído como objetivo "[...] fortalecer e ampliar atividades de pesquisa aplicada e inovação tecnológica". Assim, fortalecendo a necessidade de que haja indicadores capazes de acompanhar o desenvolvimento de tão importante tópico.

O ápice dessa investigação foi a construção de uma proposta de novos indicadores para o PDI 2019-2023, capazes de auxiliar a instituição a conquistar maior maturidade de inovação e destaque no âmbito nacional. Desse modo, foram propostos três indicadores, os quais são os seguintes: 
1) Índice de produção intelectual: Indicador que possui como objetivo mensurar a produtividade dos projetos de pesquisa. $\mathrm{O}$ cálculo desse índice relaciona o número de artigos publicados com Qualis, livros, capítulos de livros, depósitos de pedidos de patentes, patentes concedidas, registros de marca, marcas registradas, registros de programa de computador, registro de topografia de circuito integrado, desenho industrial e cultivares da Instituição no ano corrente com o total de projetos de pesquisa (exceto investigações feitas para monografias, dissertações e teses).

\section{$\frac{\text { (Total de produtos tecnológicos) }}{\text { (Total de projetos de pesquisa) }}$}

2) Índice de transferência de tecnologia: Indicador que objetiva mensurar a capacidade de transformar ativos intangíveis em inovação, fazendo com que o IF Goiano contribua com o desenvolvimento econômico regional. $\mathrm{O}$ índice relaciona o número de licença para uso de marca, cessão de marca, licença para exploração de patente, cessão de patente, licença compulsória de patente, licença para exploração de desenho industrial, cessão de desenho industrial, licença de topografia de circuito integrado, cessão de topografia de circuito integrado, licença compulsória de topografia de circuito integrado, franquia, fornecimento de tecnologia e serviço de assistência técnica e científica com o total de artigos publicados com Qualis, livros, capítulos de livros, depósitos de pedidos de patentes, patentes concedidas, registros de marca, marcas registradas, registros de programa de computador, registro de topografia de circuito integrado, desenho industrial e cultivares da Instituição no ano corrente.

\section{(Total de contratos de transferência de tecnologia)}

(Total de produtos tecnológicos)

3) Índice de produtividade dos grupos de pesquisa: Indicador que mensura o aproveitamento da capacidade de produção intelectual dos grupos de pesquisa. Esse índice utiliza como parâmetro o número de artigos publicados com Qualis, número de livros publicados, capítulos de livros, pedidos de patentes, patentes concedidas, pedidos de registro de marca, marcas registradas, registro de programa de computador, registro de topografia de circuito integrado, desenho industrial e cultivares com relação ao número de grupos de pesquisa certificados pelo CNPq que atuam no IF Goiano.

\section{(Total de produtos tecnológicos) \\ (Total de grupos de pesquisa)}

A proposta de indicadores corrobora com o disposto na Lei de criação dos Institutos Federais, Lei n. 11.892/2008, no inciso VIII, do artigo $6^{\circ}$, que dispõe da finalidade dos Institutos Federais que é "[...] realizar e estimular a pesquisa aplicada, a produção cultural, o empreendedorismo, 
o cooperativismo e o desenvolvimento científico e tecnológico". Na mesma lei, o inciso III, do artigo $7^{\circ}$, diz que são objetivos dos Institutos Federais "[...] realizar pesquisas aplicadas, estimulando o desenvolvimento de soluções técnicas e tecnológicas, estendendo seus benefícios à comunidade". A alínea do mesmo dispositivo do inciso VI reforça que os Institutos Federais têm como objetivo ministrar: "[...] e) cursos de pós-graduação stricto sensu de mestrado e doutorado, que contribuam para promover o estabelecimento de bases sólidas em educação, ciência e tecnologia, com vistas no processo de geração e inovação tecnológica".

É nesse contexto que as diversas atividades que envolvem a pesquisa, principalmente a pesquisa aplicada voltada para a inovação, que atende além do caráter acadêmico atrelado à formação na pós-graduação, busque respostas às necessidades que emergem da instituição $e$ dos anseios da sociedade. A pesquisa merece ser contemplada e tratada de maneira específica e mensurável, com indicadores de desempenho capazes de vislumbrar o progresso da instituição.

Para Speroni et al. (2015), tão importante quanto desenvolver a capacidade de fazer inovação, é a capacidade de mensuração desse processo, uma vez que disso depende a competitividade de uma empresa.

A função dos indicadores de desempenho é mostrar a ocorrência ou ausência de fatos relevantes (FRANCISCHINI; FRANCISCHINI, 2018). A relevância dos indicadores aqui sugeridos, consiste no fato de eles corroborarem com as atribuições legais da ICT no campo da inovação. Atribuições essas estabelecidas no Regulamento do NIT - IF Goiano, Resolução n. 088/2017, de $1^{\circ}$ de dezembro de 2017, e na Lei de Inovação (10.973/2004), regulamentada pelo Decreto n. 5.563, de 11 de outubro de 2005, e alterada pela Lei n. 13.243, de 11 de janeiro de 2016.

Os indicadores de desempenho só trazem informações quando houver comparação (FRANCISCHINI; FRANCISCHINI, 2018). Como critério de comparação para os indicadores aqui propostos, sugere-se a utilização de uma série histórica de dados da própria instituição, formulação de metas para cada um dos indicadores, além da análise comparativa com o cenário regional, visto que nem todos os indicadores possuem histórico.

Todo indicador e meta precisam ser monitorados e/ou controlados e, para isso, é preciso estabelecer uma periodicidade. Nesta pesquisa, as propostas de indicadores são necessárias para compor o PDI, que é um documento institucional estratégico com vigência de quatro a cinco anos. Dessa forma, não se recomenda que as avaliações dos resultados sejam feitas num período menor do que dois anos.

\subsection{Propriedade Intelectual do IF Goiano}

Com o intuito de conhecer os resultados que o IF Goiano apresenta na área de inovação, nesse tópico são apresentados dados do histórico, em alguns casos comparados com os resultados de outros IF's da Região Centro-Oeste e medidas que podem melhorar o desempenho de cada indicador proposto.

\subsection{1 Índice de Produção Intelectual}

Os Gráficos 1 e 2 mostram o histórico dos dados coletados para o cálculo do Índice de produção intelectual; é possível notar que somente em 2015 o IF Goiano começou a obter 
uma singela atuação na propriedade industrial. Em 2018, os resultados nessa área foram bem melhores, apesar da significativa queda no número de artigos publicados com Qualis.

Gráfico 1 - Número de produtos tecnológicos

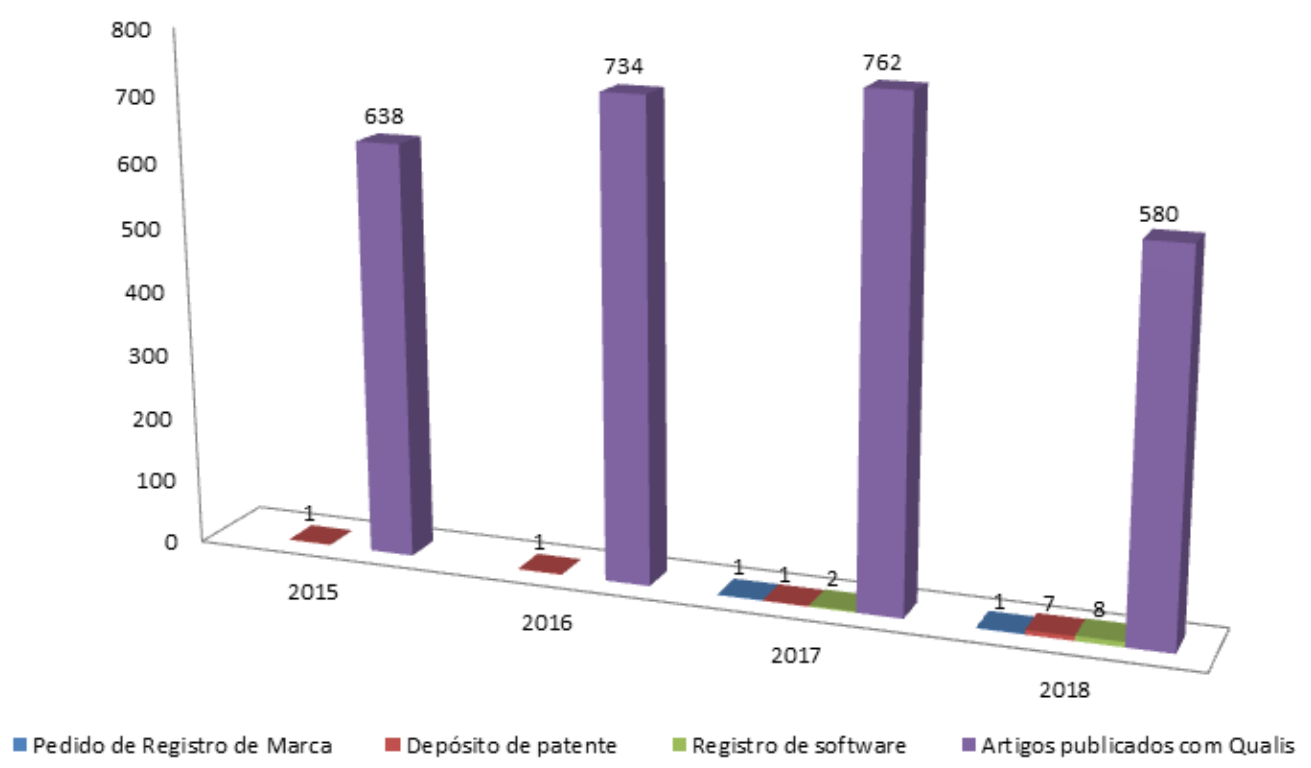

Fonte: Elaborado pelos autores deste artigo (2018)

Gráfico 2 - Número de projetos de pesquisa

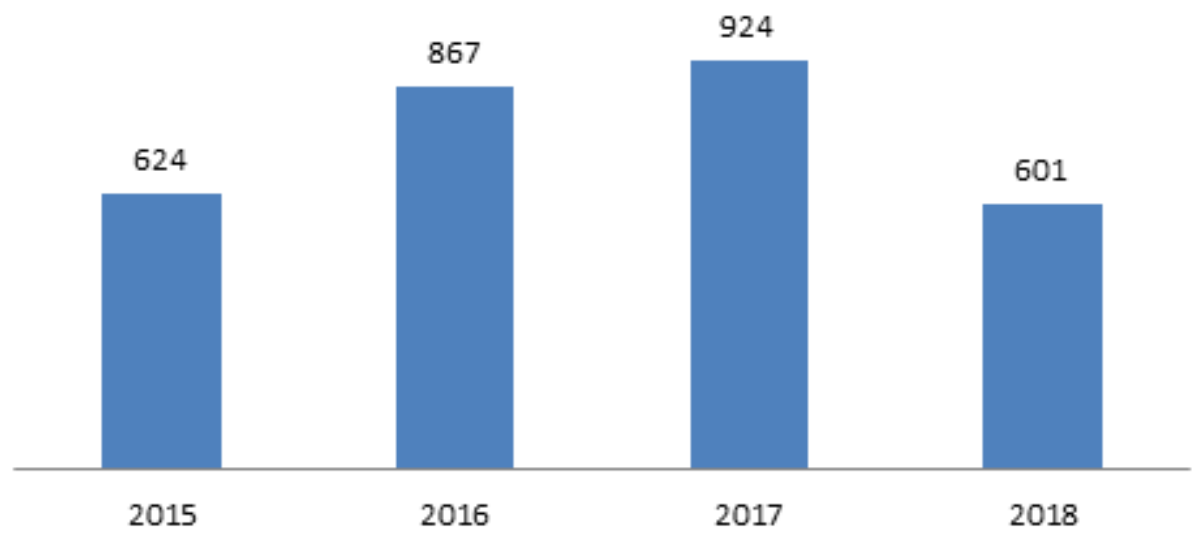

Fonte: Elaborado pelos autores deste artigo (2018)

Para fazer o cálculo do índice foi levado em conta o período de 2015 a 2018, a fórmula consiste no número de produtos tecnológicos de propriedade industrial, do ramo sui generis e artigos publicados com Qualis dividido pelo número de projetos de pesquisa do ano corrente, multiplicado por 100, conforme o Gráfico 3. 
Gráfico 3 - Índice de produtos tecnológicos dividido pelo número de projetos de pesquisa do ano corrente

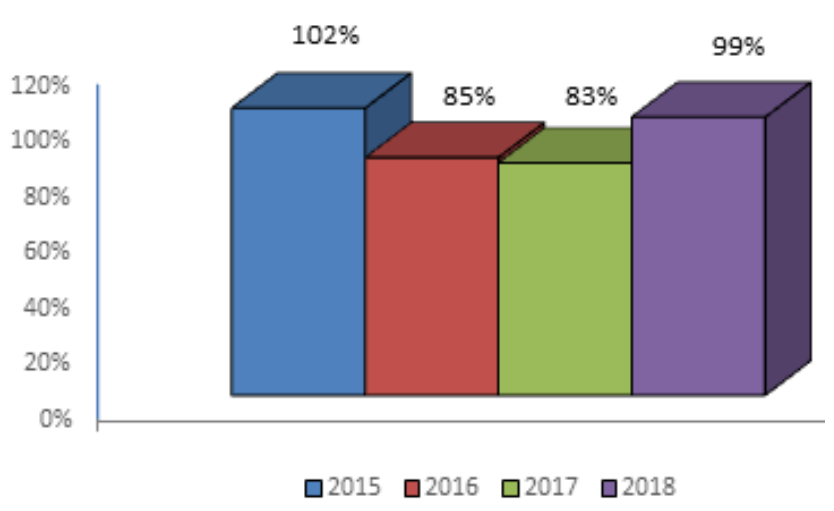

Fonte: Elaborado pelos autores deste artigo (2018)

Os resultados apresentados no Gráfico 3 evidenciam que a instituição tem explorado de forma satisfatória o seu potencial de capital humano. Contudo, faz-se necessário promover a mudança do histórico de grande produção de artigos, perante uma singela produção de outros produtos tecnológicos, principalmente ativos de propriedade intelectual, como patentes ou registro de cultivares.

É importante esclarecer que esse indicador considerou apenas os projetos de pesquisas que não são necessariamente advindos das investigações feitas em monografias, dissertações e teses. E os resultados dessas formas de pesquisa podem ter latente aspecto inovador. A inserção de disciplina de Propriedade Intelectual na grade curricular fortaleceria a cultura da inovação e contribuiria para o aumento dos números de proteções intelectuais. Tal mudança de cultura, tornaria os pesquisadores mais atentos na divulgação dos resultados obtidos em projetos de PD\&I, elaboração de acordos de confidencialidade e formulação de acordos de sigilo com parceiros externos. Além disso, a avaliação do estado da arte aliada a uma análise de roadmap para definir temas de pesquisas alavancaria ainda mais os resultados obtidos. Ressalta-se, que o NIT do IF Goiano já fez essa sugestão às Pró-Reitorias de Ensino e Pró-Reitorias de Pós-Graduação, Pesquisa e Inovação para que a disciplina de PI seja oferecida aos alunos e professores.

Para fortalecer ainda mais as práticas de inovação, o IF Goiano oferta vários editais de pesquisa. Entre eles, estão: Apoio à Produtividade em Pesquisa do Instituto Federal de Educação, Ciência e Tecnologia Goiano (PAPPE), Programa Institucional de Iniciação Científica (PIBIC) para Ensino Superior e Ensino Médio, Programa Institucional de Iniciação em Desenvolvimento Tecnológico e Inovação (PIBITI), Programa Institucional Voluntário de Iniciação Científica (PIVIC) para Ensino Superior e Ensino Médio, e, apenas em 2016, foi oferecido edital para o Programa Institucional de Apoio aos Projetos de Pesquisa em Educação do Instituto Federal de Educação, Ciência e Tecnologia Goiano (PAPED), dos quais, a maioria tem bolsas. Desse modo, tais editais podem ser considerados outra ferramenta muito eficaz para aumentar a produtividade dos pesquisadores, os quais devem ter como critérios de seleção, itens como: inovação e linhas de pesquisa que atendam as demandas da sociedade; que consequentemente, acarretem no desenvolvimento de produtos passíveis de proteção e de transferência de tecnologia. 


\subsection{2 Índice de Transferência de Tecnologia}

O INPI considera como contrato de tecnologia: licença para uso de marca, cessão de marca, licença para exploração de patente, cessão de patente, licença compulsória de patente, licença para exploração de desenho industrial, cessão de desenho industrial, licença de topografia de circuito integrado, cessão de topografia de circuito integrado, licença compulsória de topografia de circuito integrado, franquia, fornecimento de tecnologia (know-how) e serviços de assistência técnica e científica.

A importância desse indicador consiste principalmente no fato de que utilizar apenas pedidos de patentes como atividade para mensurar o nível e a qualidade da atividade tecnológica interna, apresenta inúmeras limitações, pois nem todas as patentes depositadas são concedidas, $e$ as que são concedidas, muitas delas nem chegam a ser utilizadas comercialmente no mercado. Tal situação é ainda muito comum no Brasil, ja que o retorno financeiro, obtido a partir de transferências de tecnologias, é insatisfatório, se comparado aos investimentos de PD\&I realizados. Entre outros fatores, esse baixo desempenho reflete a atual posição do Brasil no Index Global Innovation, que, em 2018, ocupou a posição de 64 lugar entre 126 países avaliados (CORNELL UNIVERSITY; INSEAD; WIPO, 2018).

É importante mencionar que não foi possível usar uma série histórica como padrão de comparação nesse índice, porque de 2010 a 2018 o IF Goiano não realizou nenhum contrato de transferência de tecnologia ou similares. Além disso, também não foi possível usar como parâmetro de comparação o cenário da Região Centro-Oeste, porque em consulta ao IFMT, IFMS, IFG e ao IFB, obteve-se a informação de que até 2018 não havia contrato algum de transferência de tecnologia com a obtenção de rendimentos. O IFMS e o IFB declararam que, ambos possuem contratos de transferências de tecnologias, contudo, nenhum deles declarou obter rendimentos.

Assim como em outros NITs, a quantidade reduzida de servidores lotados no setor de inovação pode ser um dos fatores que contribui para que esse resultado seja insatisfatório. Uma situação muito comum, existente nos NITs da Rede Federal, é que grande parte de seu quadro funcional é composto de bolsistas que trabalham por tempo determinado. Após o término de contrato, muitos deles não permanecem no quadro, fato que causa grande perda de know-how, devido ao alto índice de turnover.

A ausência de espaço físico apropriado para reuniões que necessitam de sigilo de informações, a carência de assessoria jurídica capaz de orientar e subsidiar as atividades e contratos do NIT e a ausência de orçamento específico (PIRES, 2014), também, contribuem para nutrir o círculo vicioso que impacta os resultados.

Pode ser ainda que o principal motivo de baixos índices de TT sejam explicados pelas próprias pesquisas desenvolvidas pelas ICTs, que, em geral, não estão alinhadas com as demandas do mercado que buscam resolver problemas práticos das empresas e da sociedade.

Por isso a importância de direcionar os recursos destinados à pesquisa por meio de editais que tenham critérios de inovação, uso de ferramentas como o roadmap e estudos de mercado para planejar estrategicamente o futuro dos projetos de pesquisa. Assim, acredita-se que tais ações permitirão que os produtos tecnológicos desenvolvidos sejam lançados no mercado oriundos de contratos de TT com parceiros interessados. 


\subsection{3 Índice de Produtividade dos Grupos de Pesquisa}

Para o cálculo da taxa de docentes em grupos de pesquisa foi considerado o total de professores efetivos: número de professores efetivos pertencentes a Grupos de Pesquisa (94) dividido pelo número dos professores do IF Goiano em qualquer regime (750), multiplicado por 100. Dessa forma, 12,53\% do total de docentes do IF Goiano estão engajados em grupos de pesquisa certificados pelo CNPq.

Nos grupos de pesquisa, verificou-se que as áreas de maior predominância são: Agronomia, Química, Educação, Ciência e Tecnologia de Alimentos, Ciência da Computação e Zootecnia. A localização privilegiada de alguns campi favorece o potencial desses grupos, como é o caso de Rio Verde, Urutaí e Morrinhos que justifica a maior incidência das áreas de Agronomia, Ciência e Tecnologia de Alimentos e Zootecnia.

Identificadas às áreas de conhecimento em que o IF Goiano tem maior aptidão para desenvolver PD\&I, torna-se viável e necessário desenvolver estudos de prospecção tecnológica e de inteligência competitiva no campo da propriedade intelectual, de forma a orientar as ações de inovação da instituição. Por isso sugere-se que seja feito roadmap, a partir de plataformas de prospecção tecnológica, para orientar as ações de todos os envolvidos nos projetos de PD\&I com o objetivo de consolidar e alavancar a cultura da inovação no IF Goiano, para assim direcionar de forma mais eficiente a pesquisa aplicada e atender demandas do mercado, por exemplo, encomendas tecnológicas.

A partir de uma comparação entre as instituições da Rede Federal no cenário da região Centro-Oeste, é possível verificar que o IF Goiano é a instituição que mais possui grupos de pesquisa. Consultando o CNPq, via e-SIC (BRASIL, 2019), descobriu-se que o IF Goiano tem 128 grupos de pesquisa, enquanto o IFB tem 101 grupos, o IFG tem 65 grupos, o IFMT tem 54 grupos e o IFMS tem 15 grupos.

Verifica-se, a partir do Quadro 2, que mesmo com a maior quantidade de grupos de pesquisa, o IF Goiano encontra-se em segundo lugar no ranking de produtos tecnológicos, com 10 depósitos de patentes e 10 registros de softwares.

Quadro 2 - Ativos de Propriedade intelectual no âmbito do Centro-Oeste, no período de 2008 a 2018

\begin{tabular}{|c|c|c|c|c|c|}
\hline INSTITUIÇÃo & $\begin{array}{c}\text { NúMERO DE } \\
\text { PEDIDOS DE } \\
\text { PATENTES }\end{array}$ & $\begin{array}{c}\text { NÚMERO DE REGISTROS } \\
\text { DE PROGRAMA DE } \\
\text { COMPUTADOR }\end{array}$ & $\begin{array}{c}\text { NÚMERO DE } \\
\text { REGISTROS DE } \\
\text { DESENHO INDUSTRIAL }\end{array}$ & $\begin{array}{c}\text { NúMERO DE } \\
\text { REGISTROS DE } \\
\text { CULTIVARES }\end{array}$ & $\begin{array}{c}\text { TOTAL DE } \\
\text { PRODUTOS } \\
\text { TECNOLÓGICOS }\end{array}$ \\
\hline IFB & - & 3 & - & - & 3 \\
\hline IFG & 13 & 21 & - & - & 34 \\
\hline IFMT & 4 & 4 & - & - & 8 \\
\hline IFMS & 3 & 14 & - & - & 17 \\
\hline IF Goiano & 10 & 10 & - & - & 20 \\
\hline
\end{tabular}

Fonte: Dados extraídos do e-SIC (BRASIL, 2018)

Para se fazer o cálculo do índice de produtividade dos grupos de pesquisa, foi utilizado o total de produtos tecnológicos (20) divido pelo total de grupos de pesquisa (128), multiplicado 
por 100 , cujo resultado foi de aproximadamente $15,62 \%$, percentual relativamente reduzido. O IFG, por exemplo, tem índice de produtividade dos grupos de pesquisa de $52,30 \%$.

É importante investigar porque os grupos de pesquisa do IF Goiano não conseguem melhores resultados na produção de produtos tecnológicos. Uma das causas desse reduzido desempenho pode ser a ausência de correto direcionamento das pesquisas desenvolvidas, cujos projetos de PD\&I, não estejam alinhados com as demandas do mercado. Tal situação dificulta ainda mais as parcerias com empresas.

Como já sugerido anteriormente, a utilização de processos de roadmap facilitaria as ações para incrementar a inovação, viabilizaria o desenvolvimento de pesquisas nas áreas tecnológicas em fronteiras do conhecimento. Tais medidas são mais do que necessárias para melhor utilizar os recursos humanos e financeiros envolvidos nos processos de inovação. Desse modo, espera-se que os resultados dos indicadores de inovação tenham números mais expressivos.

Outro aspecto a ser investigado é verificar se há casos em que os servidores pertencentes a esses grupos de pesquisa, por atuarem em parceria com outras instituições, estão registrando os produtos tecnológicos pela instituição parceira, sem mencionar o IF Goiano como cotitular dos ativos de PI registrados. Em vista disso, ações de divulgação e fortalecimento da política institucional de inovação visam dirimir essa possibilidade com o esclarecimento dos deveres do pesquisador para com o IF Goiano.

\section{Considerações Finais}

Com a implementação de indicadores de inovação no PDI, espera-se dar mais suporte e criar melhores condições para que o IF Goiano possa avaliar suas atividades voltadas à inovação e à pesquisa científica e tecnológica. Tais ações e iniciativas cumprem as finalidades e os objetivos estabelecidos no artigo $7^{\circ}$ da Lei n. 11.892/2008 de criação dos Institutos Federais, que estão relacionadas com o desenvolvimento de pesquisas aplicadas, desenvolvimento de soluções técnicas e tecnológicas.

O uso da estratégia de definir indicadores de desempenho personalizados, para inclui-los no PDI, possibilita o fortalecimento da política de inovação no IF Goiano. Além de contribuir com a construção de propostas de ações que ajudem a otimizar os recursos aplicados em projetos de PD\&I, práticas de sensibilização da cultura da inovação, automatização de sistema de acompanhamento e o uso de plataformas de gestão tecnológica, que poderiam influenciar positivamente no resultado efetivo a médio e longo prazo, dando mais tangibilidade à atuação do NIT.

É importante ressaltar que dos três novos indicadores propostos aqui, dois foram aprovados para compor o PDI do IF Goiano no período de 2019-2023: o índice de produção intelectual e o índice de transferência de tecnologia. A inserção de tais indicadores tem como propósito prover dados e reflexões para o amadurecimento e o desenvolvimento da política de inovação no âmbito da gestão institucional, possibilitando a estruturação e a organização dos processos que envolvem propriedade intelectual.

Desse modo, considera-se que o objetivo desta pesquisa foi conquistado e espera-se que estudos futuros avaliem, acompanhem e divulguem os impactos dessa ação proposta. 


\section{Referências}

BARNEY, J. B.; HESTERLY, W. S. Strategic Management and Competitive Advantage: Concepts and Cases. Pearson: Harlow, 2006.

BRASIL. Ministério do Planejamento, Orçamento e Gestão (MPOG). Indicadores de programas: Guia Metodológico. Brasília: MP, 2010.

BRASIL. Lei n. 10.973, de 2 de dezembro de 2004.

BRASIL. Lei n. 11.892, de 29 de dezembro de 2008.

BRASIL. Lei n. 13.243, de 11 de janeiro de 2016.

BRASIL. Sistema Eletrônico do Serviço de Informações ao Cidadão (e-SIC). [2018].

Disponível em: https:/esic.cgu.gov.br/sistema/Pedido/DetalhePedido.aspx?id=35U7+Ly1URk=. Acesso em 20 dez. 2018.

BRASIL. Sistema Eletrônico do Serviço de Informações ao Cidadão (e-SIC). [2019].

Disponível em: https://esic.cgu.gov.br/sistema/Pedido/DetalhePedido.aspx?id=Pr25xDd9uog=. Acesso em: 25 fev. 2019.

CAMILLO, E. V. et al. Indicadores de Resultado Aplicados à Indústria: um exercício a partir dos Dados do Índice Brasil de Inovação. In: XII SEMINARIO LATINO - IBEROAMERICANO DE GESTIÓN TECNOLÓGICA, ALTEC, 26-28 setembro de 2007. Anais [...], setembro de 2007.

CARAYANNIS, E. G.; CAMPBELL, D. F. J. Triple Helix, Quadruple Helix and Quintuple Helix and How do Knowledge, Innovation and the Environment Relate to Each Other? A Proposed Framework for a Trans-disciplinary Analysis of Sustainable Development and Social Ecology. International Journal of Social Ecology and Sustainable Development, [S.l.], v. 1, n. 1, p. 41-69, 2010. CGEE - CENTRO DE GESTÃO E ESTUDOS ESTRATÉGICOS. Indicadores de resultado da inovação. Brasília: CGEE, 2015.

CORNELL UNIVERSITY; INSEAD; WIPO. The Global Innovation Index 2018: Energizing the World with Innovation. Ithac, Fontainebleau and Geneva, 2018.

DRUCKER, P. F. Fator humano e desempenho: o melhor de Peter Drucker sobre administração. São Paulo: Pioneira, 1981.

EMBRAPII - EMPRESA BRASILEIRA DE PESQUISA E INOVAÇÃO INDUSTRIAL. Manual de Operação das Unidades EMBRAPII. Brasília: EMBRAPII, 2017.

ETZKOWITZ, H.; LEYDESDORFF, L. The Triple Helix as a model for innovation studies. Science and Public Policy, [S.l.], v. 25, n. 3, p. 195-203, 1998.

FRANCISCHINI, A. S. N.; FRANCISCHINI, P. G. Indicadores de Desempenho: dos objetivos à ação - métodos para elabora KPIs e obter resultados. Rio de Janeiro: Alta Books, 2018.

FREEMAN, C.; SOETE, L. Developing science, technology and innovation indicators: What we can learn from the past. Research Policy, [S.l.], v. 38, n. 4, p. 583-589, 2009.

IPEA - INSTITUTO DE PESQUISA ECONÔMICA APLICADA. Políticas de apoio à inovação tecnológica no Brasil: avanços recentes, limitações e propostas de ações. Organizadores: Lenita Maria Turchi, José Mauro de Morais. Brasília: IPEA, 2017. 
IFCE - INSTITUTO FEDERAL DE EDUCAÇÃO, CIÊNCIA E TECNOLOGIA DO CEARÁ. Plano de Desenvolvimento Institucional 2014-2018. Pró-Reitoria de Administração e Planejamento. 2014. Disponível em: https://ifce.edu.br/camocim/eventos/arquivos/pdi-instituto-federal-do-ceara.pdf/view. Acesso em: 15 maio 2018.

IFES - INSTITUTO FEDERAL DE EDUCAÇÃO, CIÊNCIA E TECNOLOGIA DO ESPÍRITO SANTO. Plano de Desenvolvimento Institucional 2014-2018. Pró-Reitoria de Desenvolvimento Institucional. 2014. Disponível em: https:/www.ifes.edu.br/images/stories/files/documentos_ institucionais/pdi_2-08-16.pdf. Acesso em: 29 maio 2018.

IF GOIANO - INSTITUTO FEDERAL DE EDUCAÇÃO, CIÊNCIA E TECNOLOGIA GOIANO. Plano de Desenvolvimento Institucional 2014-2018. Pró-Reitoria de Desenvolvimento Institucional. 2014. Disponível em: https:/www.ifgoiano.edu.br/home/images/RV/Direcao-Geral/ PDI_IFGoiano-2014-2018_12-05-2015.pdf. Acesso em: $1^{\circ}$ mar. 2018.

IF GOIANO - INSTITUTO FEDERAL DE EDUCAÇÃO, CIÊNCIA E TECNOLOGIA GOIANO. Plano de Desenvolvimento Institucional 2019-2023. Pró-Reitoria de Desenvolvimento Institucional. 2019. Disponível em: https://www.ifgoiano.edu.br/home/index.php/pdi-2019-2023.html. Acesso em: 10 abr. 2018.

IFMG - INSTITUTO FEDERAL DE EDUCAÇÃO, CIÊNCIA E TECNOLOGIA DE MINAS GERAIS. Plano de Desenvolvimento Institucional 2014-2018. Pró-Reitoria de Administração e Planejamento. 2014. Disponível em: https://www.ifmg.edu.br/portal/downloads/resolucao-019-2014anexo-pdi-2014-2018_versao-final_revisado_02_07_2014.pdf. Acesso em: 15 maio 2018.

IFPB - INSTITUTO FEDERAL DE EDUCAÇÃO, CIÊNCIA E TECNOLOGIA DA PARAÍBA. Plano de Desenvolvimento Institucional 2015-2019. Pró-Reitoria de Desenvolvimento Institucional e Interiorização 2014. Disponível em: https://www.ifpb.edu.br/praf/assuntos/documentos-praf/area6/ plano-de-desenvolvimento-institucional-pdi-2015-2019.pdf. Acesso em: 17 maio 2018.

IFSC - INSTITUTO FEDERAL DE EDUCAÇÃO, CIÊNCIA E TECNOLOGIA DE SANTA CATARINA. Plano de Desenvolvimento Institucional 2015-2019. Pró-Reitoria de Desenvolvimento Institucional. 2017. Disponível em: http://pdi.ifsc.edu.br/files/2017/04/Plano-de-DesenvolvimentoInstitucional-PDI-2015-2019_REVISADO.compressed.pdf. Acesso em: 16 maio 2018.

OCDE - ORGANIZATION FOR ECONOMIC CO-OPERATION AND DEVELOPMENT. Frascati Manual: Proposed Standard Practice for Surveys on Research and Experimental Development. Paris, 2002.

OCDE - ORGANIZATION FOR ECONOMIC CO-OPERATION AND DEVELOPMENT. Manual de Oslo. Proposta de diretrizes para coleta e interpretação de dados sobre inovação tecnológica. 3. ed. Rio de Janeiro: Finep, 2006.

PIRES, E. A. Mapeamento das estratégias para intensificar a proteção da propriedade intelectual e a transferência de tecnologia: um estudo de caso da Universidade Federal do Recôncavo da Bahia. 2014. 131 p. Dissertação (Mestrado) - Universidade Federal de Sergipe, São Cristóvão, 2014.

ROSINI, A. M.; PALMISANO, Â. Administração de sistema de informação e a gestão do conhecimento. São Paulo: Pioneira Thomson, 2003.

SCHUMPETER, J. A. The theory of economic development. Cambridge: Harvard University Press, 1934. 
SCHUMPETER, J. A. Teoria do desenvolvimento econômico. São Paulo: Abril Cultural, 1982.

SILVA, F. G.; HARTMAN, A.; REIS, D. R. Avaliação do nível de inovação tecnológica nas organizações: desenvolvimento e teste de uma metodologia. Revista Produção Online, [S.l.], v. 8, n. 4, 2008. Disponível em: http://producaoonline.org.br/index.php/rpo/article/viewFile/139/221. Acesso em: 10 jul. 2018.

SPERONI, R. M. et al. Estado da arte da produção científica sobre indicadores e índices de Inovação. Revista de Administração e Inovação, São Paulo, v. 12, n. 4 p. 49-75, out.-dez. 2015. Disponível em: http://www.revistas.usp.br/rai/article/view/101360. Acesso em: 13 ago. 2018.

STAL, E.; CAMPANÁRIO, M. A. Empresas multinacionais de países emergentes: o crescimento das multilatinas. Economia Global e Gestão, [S.l.], v. 15, n. 1, p. 55-73, 2010.

TIGRE, P. B. Gestão da inovação: a economia da tecnologia no Brasil. Rio de Janeiro: Elsevier, 2006.

TOIVONEN, M.; TUOMINEN, T. Emergence of innovations in services. Service Industries Journal, [S.l.], v. 29, n. 7, p. 887-902, 2009.

VAN DE VEN, A. H. et al. The innovation journey. New York: Oxford University Press, 1999.

VELHO, L. M. S. Estratégias para um sistema de indicadores em C\&T no Brasil. Parcerias

Estratégicas, [S.l.], n. 13, 2001.

\section{Sobre os Autores}

\section{Bruna de Oliveira Santos}

E-mail: bruna.santos@ifgoiano.edu.br

Mestra em Propriedade Intelectual e Transferência de Tecnologia para Inovação pela Universidade de Brasília em 2019.

Endereço profissional: Instituto Federal de Educação, Ciência e Tecnologia Goiano, IF Goiano, Rua 88, n. 310, St. Sul, Goiânia, GO. CEP: 74085-010.

\section{Paulo Gustavo Barboni Dantas Nascimento}

E-mail: pbarboni@unb.br

Doutor em Química pela Universidade de São Paulo em 2005.

Endereço profissional: Universidade de Brasília, UnB, Campus Ceilândia. QNN14 - Área Especial, Ceilância Sul,

Brasília, DF. CEP: 72220-140. 